\title{
EOP: ¿Es eficaz la vacunación contra rotavirus en la prevención de diarreas severas en niños?
}

\section{Escenario}

Un médico pediatra efectúa el control de salud a un niño de 2 meses de vida, al indicarle las inmunizaciones correspondientes, la madre le comenta haber visto una nota en un programa de televisión acerca de la efectividad de una nueva vacuna contra la infección por rotavirus y le pregunta si su niño debería recibirla.

Si bien el médico está al tanto de que dicha vacuna aún no está disponible en forma comercial en nuestro medio (sí en el contexto de ensayos clínicos), se pregunta qué impacto tendría la utilización de la inmunización activa en el curso de la infección por rotavirus en los lactantes sanos.

\section{Pregunta}

¿En los lactantes sanos menores de 1 año (población), la administración de la vacuna humana a virus vivos atenuados RIX 4414 (intervención), reduce el número de episodios de diarrea severa por rotavirus o la mortalidad a causa de los mismos (resultado)?

\section{Gastroenteritis Aguda por Rotavirus}

La infección por rotavirus es la principal causa de gastroenteritis severa en los niños en todo el mundo. Anualmente se estima que ocasiona 870.000 muertes y 2 millones de hospitalizaciones entre los niños menores de 5 años. La mortalidad y requerimientos de internación entre los niños varían significativamente dependiendo de la accesibilidad al sistema de salud en los países en desarrollo. Los factores sanitarios ambientales no parecen estar relacionados con la ocurrencia de diarrea por rotavirus, lo cual explicaría la similar incidencia de esta patología en países desarrollados y en vías de desarrollo., ${ }^{2,3}$ Los casos clínicamente evidentes se producen entre los 4 meses y los 2 años de edad (más de $80 \%$ de los casos se presentan en el primer año de vida y $35 \%$ antes de los 6 meses de edad en los países en desarrollo), ocasionalmente ocurren casos en lactantes menores de 3 meses, quienes estarían virtualmente protegidos por el pasaje de anticuerpos por vía transplacentaria y por la leche materna. En nuestro país se estima que el $20 \%$ de las internaciones por diarrea se deben a infecciones por rotavirus (alcanzando cifras cercanas al $50 \%$ en los meses invernales), y entre estos casos, el $71 \%$ corresponden a menores de un año. ${ }^{1,4}$ Hacia los 3 años de edad casi todos los niños han estado en contacto con el virus y aunque la inmunidad mucosa no es completamente eficaz para prevenir nuevas infecciones, luego de un episodio alcanza el $77 \%$ y frente a episodios severos, la inmunidad natural confiere una protección del 87 al 100\% luego de una o dos infecciones respectivamente. ${ }^{2}$ En ese contexto, una nueva vacuna podría causar una disminución muy marcada del número de consultas médicas y hospitalizaciones por diarreas infantiles y podría reducir el número de defunciones asociadas a las mismas en los países en desarrollo. En 1998, la FDA autorizó la administración oral de la vacuna tetravalente antirrotavirus rhesus (RRV-TV, RotaShield). Varios estudios realizados en los EE.UU., Finlandia y Venezuela han encontrado que la RRV-TV es muy eficaz para la prevención de la enfermedad por rotavirus. Sin embargo, otros estudios realizados en Perú y Brasil presentaron resultados inconsistentes, con una eficacia mucho menor. Desde la introducción de la RRV-TV, se informaron varios casos de intususcepción después de la administración de la vacuna, lo que contribuyó a su retiro meses más tarde. Las vacunas actualmente en estudio en países latinoamericanos son las denominadas vacunas a virus vivos atenuados, humanas, de administración oral (RIX 4414, 89-12), y a ellas se refirió fundamentalmente la presente búsqueda.

\section{Estrategia de la Búsqueda}

Se realizó una búsqueda en Medline utilizando los filtros metodológicos (Clinical Queries) para estudios de terapéutica y revisiones sistemáticas utilizando como palabras clave los términos MESH "rotavirus vaccine", limitando la búsqueda a los 5 últimos años. La búsqueda arrojó 21 citas, de las cuales 2 parecían relevantes para responder la pregunta.

\section{Resumen de la evidencia}

Soares-Weiser K, Goldberg E, Tamimi G. Rotavirus vaccine for preventing diarrhoea (Cochrane Review). In: The Cochrane Library, Issue 2, 2005. Oxford: Update Software.
Objetivos: Evaluar las vacunas antirrotavirus en relación con la prevención de la diarrea, muerte y acontecimientos adversos por rotavirus.

Fuente de Datos: Registro de ensayos del Grupo Cochrane, EMBASE, LILACS, Biological Abstracts, listas de referencias de los artículos y contacto con los investigadores y fabricantes de vacunas antirrotavirus.

Selección de estudios y extracción de datos: Ensayos controlados aleatorizados que compararan la eficacia de las distintas vacunas antirrotavirus (Vacuna de virus vivos atenuados bovina, RIT4237, WC3, W179-9, QHBRV; Vacuna antirrotavirus rhesus, RRV, RRV-TV; Vacuna humana de virus atenuados, M37, 89-12; Otras vacunas antirrotavirus, BIRVI, RV de Cordero) contra placebo 0 ninguna intervención en niños y adultos. Fueron elegibles 64 ensayos publicados hasta el año 2003.

Resultados Principales: En cuanto a la prevención de los episodios de diarrea por rotavirus, los tres tipos de vacunas analizadas presentaron una eficacia significativa que varió entre el $41 \%$ para las vacunas rhesus y el $68 \%$ para la cepa humana atenuada $89-12$. Las distintas vacunas también mostraron un beneficio sumamente significativo en la prevención de episodios graves de infección por rotavirus, así como de aquéllos que requirieron hospitalización. Al analizar el impacto de la vacunación en la ocurrencia de diarrea por cualquier agente etiológico, se encontró un efecto beneficioso de las vacunas rhesus y de las vacunas bovinas y ninguna diferencia estadísticamente significativa para las vacunas humanas.

Se registraron 57 casos de muertes por todas las causas entre los diferentes ensayos, sin embargo debido a insuficiente información en la mayoría de los estudios, el metanálisis sólo fue posible en cuatro ensayos de vacunas antirrotavirus rhesus, con un total de diez muertes en 6029 niños. Los datos combinados no indicaron una diferencia estadísticamente significativa entre los niños asignados al azar para recibir vacunas rhesus o placebo en el número de muertes (RR 0,72 [IC95\% 0,16-3,12]). Cuarenta y nueve ensayos proporcionaron información sobre la seguridad de las vacunas antirrotavirus en 16.602 niños. Fue posible realizar un metanálisis para tres estudios de las vacunas antirrotavirus rhesus con un total de 4123 niños en cuanto a la ocurrencia de casos de intususcepción, sin embargo, los datos combinados no indicaron una diferencia estadísticamente significativa en el número de casos entre los niños asignados al azar a recibir vacunas rhesus o placebo. En cuanto a los eventos adversos menores, la fiebre ocurrió más frecuentemente entre los niños que recibieron vacunas rhesus (RR 2, [IC95\% 1,51-2,64]) y no hubo diferencia significativa con el placebo en el caso de las vacunas bovinas y humanas.

Tabla 1: Eficacia de las distintas vacunas antirrotavirus

\begin{tabular}{|c|c|c|c|c|c|c|c|c|c|}
\hline & \multicolumn{3}{|c|}{ Vacunas Phesus } & \multicolumn{3}{|c|}{ Vacunas Bovinas } & \multicolumn{3}{|c|}{ Vacunas Humanas } \\
\hline & BR (LE $95 \%)$ & RRR & WVIT & BR (IC $95 \%$ ) & Bin & WVIT & BR (E) $95 \%$ ) & RiR & WWT \\
\hline Diarrea por Rotavirus & $0,59(0,50-0,70)$ & $41 \%$ & 19 & $0,59(0,45-0,76)$ & $41 \%$ & & $0,32(0,23-0,45)$ & $68 \%$ & 11 \\
\hline Episodios Graves & $0,42(0,31 \cdot 0,57)$ & $58 \%$ & 18 & $0,38(0,24-0,60)$ & $62 \%$ & 15 & $0,21(0,13-0,35)$ & $79 \%$ & 14 \\
\hline Hospitalización & $0,48(0,27-0,86)$ & $52 \%$ & & $0,37(0,18-0,74)$ & $63 \%$ & 55 & $0,21,(0,09-0,48)$ & $79 \%$ & 51 \\
\hline Diarrea por todas las causas & $0,86(0,80-0,92)$ & $14 \%$ & 11 & $0,73(0,60-0,89)$ & $27 \%$ & 11 & $0,91(0,57-1,44)$ & * & \\
\hline Mortalidad todas las causas & $0,72(0,16-3,12)$ & * & & Sin datos & & & Sin datos & & \\
\hline Intususcepción & $0,42(0,07-2,42)$ & * & & Sin datos & & & Sin datos & & \\
\hline
\end{tabular}

${ }^{*}$ No significativo

Conclusión de los revisores: Los tres tipos de vacunas antirrotavirus analizadas presentaron altos niveles de eficacia en la prevención de diarrea por rotavirus y diarrea por todas las causas. En cuanto al impacto sobre la gravedad de los episodios si bien se reportó una eficacia significativa, se detectó un nivel elevado de heterogeneidad entre los estudios (principalmente entre las distintas vacunas rhesus y bovinas), lo cual dificulta la interpretación de los datos. Las pruebas acerca de la seguridad y el impacto sobre la mortalidad asociada a gastroenteritis y deshidratación son escasas y no concluyentes, por lo que son necesarios nuevos estudios que permitan valorar estos resultados. No se demostró interferencia de la administración concomitante de DPT y OPV en la eficacia de las vacunas.

Salinas B, Perez Schael I, Linhares A. Evaluation of Safety, Immunogenicity and Efficacy of an Attenuated Rotavirus Vaccine, RIX 4414. A Randomized, Placebo-Controlled Trial in Latin 
American Infants. Pediatric Infectious Disease Journal 2005; 24 : 807-16.

Objetivo: Documentar el perfil de inmunogenicidad, seguridad y eficacia de la vacuna humana a virus vivos atenuados, de administración oral RIX 4414, en tres distintas dosificaciones.

Diseño: Ensayo aleatorizado, doble ciego, controlado por placebo. Lugar de realización: Áreas urbanas de bajos y medianos ingresos en 3 países latinoamericanos: Brasil, Méjico y Venezuela.

Población: Participaron del estudio 2155 lactantes sanos, sin patología perinatal, de entre 6-12 semanas de vida. Se excluyeron niños con antecedente de haber padecido infección previa por rotavirus, inmunocompromiso o enfermedades que comprometieran el sistema digestivo.

Intervención: Se aleatorizó a cuatro ramas con una proporción 1:1:1:1, tres grupos correspondieron a diferentes dosificaciones de la vacuna humana a virus vivos atenuados cepa RIX 4414, administrada por vía oral a los 2 y 4 meses de vida; el cuarto grupo correspondió a los controles, quienes recibieron 2 dosis de placebo (2 y 4 meses), de constitución idéntica a la vacuna excepto por el principio activo. Tanto los pacientes como los médicos se mantuvieron ciegos a la intervención. El seguimiento se efectuó hasta el año de vida y fue completado por el $93 \%$ de los niños.

Mediciones: Con el objeto de valorar la inmunogenicidad se efectuaron dosajes de $\lg A$ antirrotavirus al inicio del estudio a fin de documentar el estado pre vacunación, y posteriormente en un subgrupo de niños a los 2 meses de recibir la primera y segunda dosis, y a todo el grupo al completar el seguimiento, al año de vida. Los padres registraron en cartillas la ocurrencia de síntomas considerados reacciones adversas potenciales, $u$ otros no preespecificados durante 15 días posteriores a cada dosis. Los eventos adversos serios se registraron durante todo el período de estudio.

Se efectuó vigilancia (visitas semanales) a cargo de agentes sanitarios para detectar y llevar a cabo el seguimiento de los casos de gastroenteritis, la gravedad de los mismos se valoró mediante un puntaje clínico que consideró el número de deposiciones y vómitos por día, la presencia de fiebre, deshidratación, requerimientos de hospitalización y duración del episodio. En los casos de gastroenteritis detectados, el diagnóstico etiológico se basó en la detección antigénica mediante ELISA en materia fecal, con confirmación de los casos positivos con PCR.

Resultados Principales: El análisis de seguridad se efectuó por intención de tratar, no se registraron diferencias significativas en la incidencia de reacciones adversas en los 15 días posteriores a la vacunación en los distintos grupos. Se consigna la ocurrencia de eventos adversos serios en el $10 \%$ de los participantes (sin diferencia entre los grupos intervención y control), aunque no se reporta la naturaleza de dichos eventos. Ocurrieron 3 muertes durante el seguimiento ( 1 en el grupo placebo y 2 en el de vacunación), aparentemente sin relación con la intervención. Valoración de la inmunogenicidad, se detectó seroconversión $(\lg A)$ en $38 \%$ al $43 \%$ de los niños 2 meses después de la primera dosis y en 61 al $65 \%$ 2 meses después de la segunda dosis. Al concluir el período de seguimiento, el $73-77 \%$ de los niños vacunados y $41 \%$ de los no vacunados presentaban IgA antirrotavirus.

Valoración de la Eficacia: El análisis incluyó a los niños que recibieron 2 dosis de vacuna o placebo y que no presentaron episodios de gastroenteritis hasta 2 meses después de la segunda dosis ( $85,7 \%$ del total, $86 \%$ del grupo intervención y 84,5 del grupo control). Si bien los autores refieren que al comparar los datos del análisis efectuado con los del total de la cohorte (intención de tratar) no se hallaron diferencias significativas, no consignan tales datos en la publicación. La eficacia se valoró para la prevención de un episodio de diarrea por rotavirus, episodios severos según la escala de valoración clínica y episodios que requirieron hospitalización. La eficacia se valoró para la prevención de un episodio de diarrea por rotavirus, episodios severos según la escala de valoración clínica y episodios que requirieron hospitalización.

Tabla 2: Indicadores de efectividad de la vacuna antirrotavirus.

\begin{tabular}{c|c|c|c|c} 
& RPR (IC 95\%) & RPA (IC 95\%) & NiIT (IC 95\%) & P \\
\hline Diarrea por Rotavirus & $63 \%(47-74)$ & $7 \%(4-10)$ & $14(10-25)$ & $<0,0001$ \\
\hline Episodios severos & $74 \%(57-84)$ & $5,5 \%(3-8)$ & $18(12-33)$ & $<0,0001$ \\
\hline $\begin{array}{c}\text { Episodios que requieren } \\
\text { hospitalización }\end{array}$ & $79 \%(52-91)$ & $2,4 \%(0,8-4,1)$ & $41(24-125)$ & $<0,0001$ \\
\hline
\end{tabular}

Conclusión de los autores: La administración temprana (2-4 meses) de 2 dosis orales de la vacuna antirrotavirus RIX 4414 mostró ser altamente efectiva en la prevención de episodios de diarrea por rotavirus, incluyendo los episodios graves y los que ocasionaron hospitalización.

Fuente de Financiación y conflictos de interés: GlaxoSmithKline Biologicals, Rixensart, Belgium.

\section{Comentario}

La disponibilidad de una vacuna contra rotavirus representa una promesa en el corto plazo. De acuerdo a la información aquí revisa$\mathrm{da}$, los ensayos realizados hasta el momento tuvieron suficiente poder estadístico para confirmar la eficacia de las distintas vacunas en la prevención de la infección por rotavirus, pero falta aún aclarar el impacto en la mortalidad causada por las mismas en la población de niños pequeños. En este sentido, en ambos estudios presentados los niños se encontraron bajo supervisión rigurosa efectuándose diagnóstico temprano y seguimiento cuidadoso de los casos de diarrea, situación por demás artificial, en especial en los países en desarrollo, que por otro lado son los que presentan la mayor carga de la enfermedad y sus complicaciones (deshidratación, desnutrición y mortalidad). Además, la mayoría de los ensayos probaron la vacuna en los individuos de bajo riesgo; sin embargo, la morbilidad y mortalidad mayor ocurre en los niños de alto riesgo, en particular en los niños desnutridos. Si bien los tres grupos de vacunas reportaron altos niveles de eficacia en cuanto a la prevención de episodios graves de infección por rotavirus, la gravedad de los mismos se valoró mediante diferentes escalas y quizás no represente el mismo espectro de gravedad de la enfermedad. Asimismo, una vez más la supervisión rigurosa de los niños, quizás haya influenciado la baja frecuencia de diarrea grave medida mediante hospitalización y deshidratación. Es probable que las cuestiones planteadas previamente sólo puedan aclararse luego de la implementación de la vacuna en el contexto poblacional, dentro de la realidad sanitaria, nutricional y de accesibilidad al sistema de salud de nuestros niños. Sin embargo, antes de lograrse la inclusión de esta nueva vacuna en los programas de inmunización, deberá probar ser costoefectiva con respecto a las alternativas (relativamente de bajo costo) del manejo de la diarrea y deshidratación. A pesar del gran número de estudios realizados, persiste aún la incertidumbre relativa a la seguridad de las vacunas, lo cual deriva principalmente de déficits metodológicos y falta de claridad en el reporte de eventos adversos. Este punto podría ser resuelto por los ensayos en curso, en poblaciones más amplias. En cuanto a la recomendación en casos individuales parecería razonable considerar el riesgo del niño de contraer la infección a una edad temprana, y de presentar complicaciones a causa de la misma, situaciones que son de menor frecuencia entre niños sanos de buen nivel socioeconómico con garantía de acceso oportuno al sistema de salud.

Silvia Clelia Chaves [ Médica Pediatra. Hospital Materno Infantil de San Isidro Carlos Gianantonio. ]

\section{Referencias}

1. Gomez J, Nates S, De Castagnaro N. Anticipating rotavirus vaccines: review of epidemiologic studies of rotavirus diarrhea in Argentina. Rev Panam Salud Pública/Pan Am J Public Health 1998;3(2):69-77.

2. Matson D. Prevention and treatment of viral gastroenteritis in children. In: UpToDate 13.2. Disponible en http://www.uptodate.com

3. Sabbaj L, De Petre E, Gomez J. Rotavirus en la diarrea aguda. Archivos argentinos de pediatría 2001; 99(6):486-490.

4. Alonso M. Gastroenteritis por rotavirus. Archivos argentinos de pediatría 2001; 99(6):483-484.

5. Soares-Weiser K, Goldberg E, Tamimi G. Rotavirus vaccine for preventing diarrhoea (Cochrane Review). In: The Cochrane Library, Issue 2, 2005. Oxford: Update Software.

6. Salinas B, Perez Schael I, Linhares A. Evaluation of Safety, Immunogenicity and Efficacy of an Attenuated Rotavirus Vaccine, RIX 4414. A Randomized, 